\title{
An Alternative Manifold for Cosmology Using Seifert Fibered and Hyperbolic Spaces
}

\author{
Maria E. Mejía1, Reinaldo R. Rosa ${ }^{2}$ \\ ${ }^{1}$ Departamento de Matemáticas, Universidad de Guadalajara, Guadalajara, México \\ ${ }^{2}$ Laboratório Associado de Computação e Matemática Aplicada, Instituto Nacional de Pesquisas Espaciais, São \\ José dos Campos, Brasil \\ Email: mariesther.mejia@gmail.com, reinaldo.rosa@pq.cnpq.br
}

Received 9 November 2013; revised 18 December 2013; accepted 29 December 2013

Copyright (C) 2014 by authors and Scientific Research Publishing Inc.

This work is licensed under the Creative Commons Attribution International License (CC BY). http://creativecommons.org/licenses/by/4.0/

(c) (i) Open Access

\begin{abstract}
We propose a model with 3-dimensional spatial sections, constructed from hyperbolic cusp space glued to Seifert manifolds which are in this case homology spheres. The topological part of this research is based on Thurston's conjecture which states that any 3-dimensional manifold has a canonical decomposition into parts, each of which has a particular geometric structure. In our case, each part is either a Seifert fibered or a cusp hyperbolic space. In our construction we remove tubular neighbourhoods of singular orbits in areas of Seifert fibered manifolds using a splice operation and replace each with a cusp hyperbolic space. We thus achieve elimination of all singularities, which appear in the standard-like cosmological models, replacing them by "a torus to infinity". From this construction, we propose an alternative manifold for cosmology with finite volume and without Friedmann-like singularities. This manifold was used for calculating coupling constants. obtaining in this way a theoretical explanation for fundamental forces is at least in the sense of the hierarchy.
\end{abstract}

\section{Keywords}

Topology, Cosmology, Thurston's Theory, Singularity-Free

\section{Introduction}

In his field equations, Einstein just provided a compact mathematical tool that made it possible to develop a theory to describe the general configuration of matter-energy and space-time for the Universe. Nevertheless, the questions related to the global shape of space and, in particular, its finite or infinite extension, cannot be fully answered by the General Relativity (a local physical theory). That is why, the use of topology (a global mathe- 
matical theory) is necessary to construct a new manifold which could answer some of those questions. Accordingly, many topological "alternatives" of three-dimensional spaces can thus be used to build such cosmological universe models.

The investigation of topological changes may bring a new approach to studying the early cosmology of the universe [1] [2]. The problem of changing topology has been discussed from different points of view in both classical and quantum gravity [3] [4]. In addition, Gauge theory [5] provides an alternative to solve some problems that general relativity can not.

Efremov [6] proposed a manifold which admits 3-dimensional sections of Euclidean space-time cobordisms describing topology changes. In this work we take the Seifert Fibered Spaces represented by homologic spheres and we improve the idea of Efremov [6] [7] and Mitskievich [8] [9] adding hyperbolic cusp spaces. We describe the process of construction of a cosmological model which was used to calculate the coupling constants for fundamental forces as shown in [10].

We like to point out that our Model of Space-Time does not have singular orbits (those are obtained by factorization with respect to the action group $S^{1}$ ) because they have been replaced by an hyperbolic manifold. Also, comparing the coupling constants obtained in [6] with the new ones, we can see that the new model has a better fit with those obtained by experiments (see [10]).

Other works which can be a motivation for using hyperbolic manifolds in physics are: [11]-[14]. However, compared to the work presented here, their approaches are in a very different way because they are limited to a decomposition of Seifert Fibered Spaces and do not use Thurston's theory for the hyperbolic part.

First we make the decomposition of Seifert Fibered Spaces in prime spaces. This process includes a collection of cuts of a 3-dimensional space $M^{3}$ in two parts along 2-dimensional spheres such that the space $M^{3}$ is separated into two parts and neither of these is a 3-dimensional disc. This operation is the inverse of the connected sum. The result of gluing two of 3-dimensional disc to each component of the boundary is to obtain a simpler 3-manifold every time we do the process. Kneser [15] proved that such process is terminated after a finite number of steps. We call prime components of $M^{3}$ as each of the parts that were obtained as a result of this decomposition process. They are solely defined except by homeomorphisms, see [16].

After that, we involve the decomposition of cuts along a torus. The reverse operation to splice described in [17], has been discovered by Johanson [18], Jaco and Shalen [19]. This decomposition along the torus $\left(T_{i}^{2} \subset M^{3}\right)$ gives a set of 3-manifolds of which some are Seifert Fibered manifolds and others allow hyperbolic structures. Previous works [6] and [9], considered a special case. Namely, a 3-dimensional space contains only Seifert fibered spaces represented by homological spheres as parts.

Here, we extend those ideas using Thurston's conjecture (now proved) [20]-[22] and the work of Jaco [19] [23] and Johanson [18]. This allows glueing a suitable hyperbolic space in Seifert fibered space and taking the Seyfert hyperbolic invariants inside of a hyperbolic space.

\section{Introducing Seifert Manifolds and Hyperbolic Spaces}

There are several definitions in literature about Seifert Manifolds, here we introduce our own definition of Seifert Manifolds. For hyperbolic spaces we will use Thurston's theory. Then we will use all those concepts for the construction of a new Manifold representing the cosmological Universe.

\section{Seifert Manifolds}

Definition 1. A Seifert Fibered Manifold (SFM) is an orientable three-manifold $M, M$ is the collection $\left\{C_{\alpha}\right\}$ of pairwise disjoint simple closed curves, called fibers, such that for each $\alpha$ there is a closed neighbourhood $V$ of $C_{\alpha} \in M$ which is a solid torus and there is a covering map $p: D^{2} \times S^{1} \rightarrow V$ such that:

1) $p$ maps each $x \times S^{1},\left(x \in D^{2}\right)$ to $C_{\beta}$.

2) $p^{-1}\left(C_{\alpha}\right)$ are connected.

3) The covering translation group is generated by $\tau_{a, b}$ where

$$
\tau_{a, b}\left(r \mathrm{e}^{\mathrm{i} \theta}, \mathrm{e}^{\mathrm{i} \phi}\right)=\left(r \mathrm{e}^{i\left(\theta+\frac{2 a \pi}{b}\right)}, \mathrm{e}^{i\left(\phi+\frac{2 a \pi}{b}\right)}\right) .
$$

Definition 2. If $M$ is a 3-compact manifold and $M$ has a Seifert fibration, there is a smooth map 
$f: M \rightarrow S, S$ surface with the following property. Each $p \in S$ has a closed neighbourhood $U$ such that, the restriction $f$ to $f^{-1}(U)$ is a diffeomorphism from Seifert fibration to a solid torus. To be more precise, there are relatively prime integers $p$ and $q$, with $0 \leq q<p$ and the diffeomorphisms $g: U \rightarrow D^{2}$ and $h: f^{-1}(U) \rightarrow S^{1} \times D^{2}$ such that the diagram

$$
\begin{array}{ccc}
f^{-1}(U) & \underset{h}{ } & S^{1} \times D^{2} \\
\downarrow f_{\mid f^{-1}(U)} & & \downarrow f_{p, q} \\
U & \underset{g}{\rightarrow} & D^{2}
\end{array}
$$

is commutative.

Applying the previous definitions. Consider the trivial fibered cylinder $D^{2} \times I$, with fibers ${ }^{1}\{x\} \times I$. Identifying cylinder caps and making a rotation $\frac{2 \pi p}{q},(p, q \in \mathbb{Q})$ to a cylinder we obtain a fibered torus, see Figure 1.

The reader can also see [23].

See Appendix for other useful definitions.

\section{Hyperbolic Spaces}

In his notes, Thurston [21] describes different models for hyperbolic spaces. However we only take here the upper-half space model. We believe that this particular hyperbolic space has the necessary geometry in order to fulfil the requirements of the physical space. It has itself differents approaches, then in physics we want to be one part flat. In this space inasmuch we can find the ratio of the Euclidean distance. Also has the useful characteristic of having points at infinity.

The upper half-space model (taked from [21]).

This is closely related to the Poincaré disc model and the southern hemisphere, but it is often more convenient for computation or for constructing graphics. To obtain the upper-half space model, rotate the sphere $S^{n}$ in $\mathbb{R}^{n+1}$ so that the southern hemisphere lies in the half-space $x_{n} \geq 0$ is $\mathbb{R}^{n+1}$. Now, the stereographic projection from the top of $S^{n}$ (which is now on the equator) sends the southern hemisphere to the upper half-space in $\mathbb{R}^{n+1}$. As shown in Figure 2.

A hyperbolic line, in the upper half-space, is a circle perpendicular to the bounding plane $\mathbb{R}^{n+1} \subset \mathbb{R}^{n}$.

The hyperbolic metric is:

$$
d s^{2}=\left(\frac{1}{x_{n}}\right)^{2} d x^{2} .
$$

This representation of a hyperbolic space is suitable in physics because we have two things. A manifold with finite volume [24] and the Euclidean image of a hyperbolic object moving toward $\mathbb{R}^{n-1}$ has size precisely proportional to the Euclidean distance from $\mathbb{R}^{n-1}$.

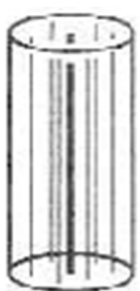

1(a)

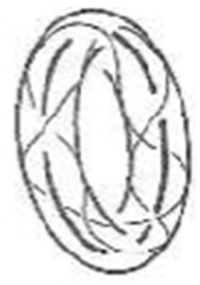

1(b)

Figure 1. (a) Represents the cilinder with fibres and 1 (b) is the fibered torus that will appear in our construction.

${ }^{1}$ The fibers play the role of a coordinate basis for objects in the field. 


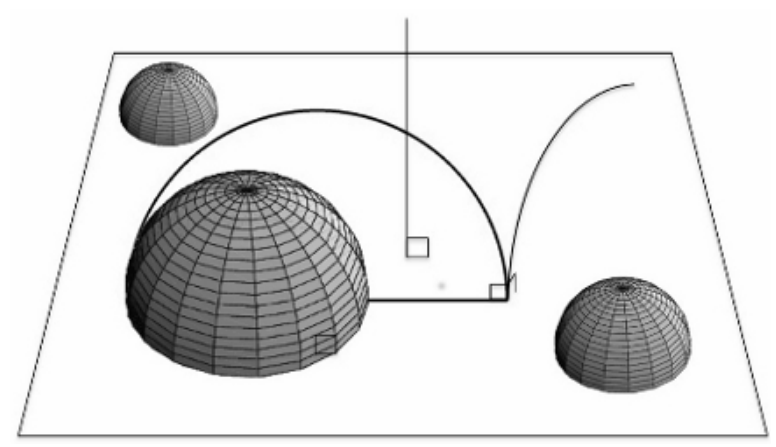

Figure 2. Upper half space model.

Here we present the triangulation of the Seifert spheres and hyperbolic spaces in order to preserve the orientation of manifolds. Those are used in [10] in order to calculate a discrete volume. After this we use the Eisenbud ideas for manifold decompositions and gluing. See [17] for a complete description of links, splice operation and their characteristics [25]. Here we only need highlight the following theorem.

Theorem 1. Jaco-Shalen, Johanson Torus Decom-position.

If $M$ is an oriented, compact, irreducible 3-manifold, then there is a finite collection of incompressible disjoint torus $T_{1}, \cdots, T_{n}$ in $M$ such that splitting $M$ along the union of those torus, produces manifolds $M_{i}$ that are either Seifert or atoroidal (every incompressible torus in $M_{i}$ is isotopic to a component toroidal of $\partial M_{i}$ ). Moreover, a minimal collection of such torus $T_{i}$ is unique under isotopy.

This theorem together with the standardization of Thurston's theorem states: there is a collection of incompressible tori $W \subset M$, unique under isotopy ambient, which cut $M$ in Seifert fibered manifolds and hyperbolic manifolds of finite volume. See [26].

In the next sections we will do the triangulation of Seifert fibered spaces as homology spheres as well as the hyperbolic space. But first, we see some definitions and constructions that will be helpful.

\section{SFH-Spheres, Derived and Primary Sequences}

Definition 3. We consider homology Brieskorn spheres $\Sigma\left(a_{1}, a_{2}, a_{3}\right)$, which are a special case of the SFHspheres (Seifert fibered homology spheres), which contain only three exceptional orbits. These SFH-spheres are the intersection of the surface

$$
z_{1}^{a_{1}}+z_{2}^{a_{2}}+z_{3}^{a_{3}}=0, \quad\left(z_{i} \in \mathbb{C}_{i}\right)
$$

in a 3-complex dimensional space with the 5-dimensional unit sphere

$$
\left|z_{1}\right|^{2}+\left|z_{2}\right|^{2}+\left|z_{3}\right|^{2}=1 \text {. }
$$

If $a_{1}, a_{2}, a_{3}$ are relatively prime and if $a_{i}>1$ then there is a unique Seifert fibration with Seiferts invariant not normalized

$$
\left(a_{1}, b_{1}\right) ;\left(a_{2}, b_{2}\right) ;\left(a_{3}, b_{3}\right)
$$

subject to

$$
\sum_{i=1}^{3} b_{i} \sigma_{i}=1
$$

where $\sigma_{i}=a / a_{i}$ and $a=a_{1} a_{2} a_{3}$.

It is possible to represent this relationship by the Euler number of the Seifert fibration of SFH-sphere $\Sigma(\underline{a}):=\Sigma\left(a_{1}, a_{2}, a_{3}\right)$ [27].

$$
e(\Sigma(\underline{a}))=\sum_{i=1}^{3} b_{i} / a_{i}=1 / a
$$

which is a topological invariant of $\Sigma(\underline{a})$. The SFH-sphere $\Sigma(\underline{a})$ may also be described as a cyclic cover 
$a_{3}$-folds of $S^{3}$, branched along the torus knot of type $K\left(a_{1}, a_{2}\right)$ [28].

\subsection{SFH-Spheres Derived}

Given a SFH-sphere $\Sigma(\underline{a}):=\Sigma\left(a_{1}, a_{2}, a_{3}\right)$ we define the derivative (in the sense that a SFH-sphere is obtained from another SFH-sphere) of a SFH-sphere, as follows:

$$
\Sigma^{(1)}(\underline{a}):=\Sigma^{(1)}\left(a_{1}, a_{2}, a_{3}\right):=\Sigma\left(a_{1}, \sigma_{1}, a+1\right) \equiv \Sigma\left(a_{1}^{(1)}, a_{2}^{(1)}, a_{3}^{(1)}\right)=\Sigma^{(1)}\left(\underline{a^{(1)}}\right),
$$

and the Seifert invariants are:

$$
\left(a_{i}^{(1)}, b_{i}^{(1)}\right)=\left\{\left(a_{1}, b_{1}\right),\left(\sigma_{1}, \delta_{1}\right),(a+1,-1)\right\},
$$

where $\delta_{1}=b_{2} a_{3}+b_{3} a_{2}, \sigma_{1}=\frac{a}{a_{1}}, a=a_{1} a_{2} a_{3}$.

It is easy to prove that the manifold in (5) with invariants (6) is a homology sphere because its Euler number satisfy (4). We have:

$$
e\left(\Sigma^{(1)}(\underline{a})\right)=\sum_{i=1}^{3} \frac{b_{i}^{(1)}}{a_{i}^{(1)}}=\frac{1}{a^{(1)}},
$$

where $a^{(1)}=a_{1}^{(1)} a_{2}^{(1)} a_{3}^{(1)}$ and $a_{1}^{(1)}, a_{2}^{(1)}, a_{3}^{(1)}$ are coprime.

Since any SFH-sphere has unique Seifert fibration, the meaning of derivative of the sphere coincides with the derived homology structure of the Seifert fibered space (i.e. acquires characteristics of SFH-spheres).

Definition 4. By induction, we define the derivative $\Sigma^{(\ell)}(\underline{a})=\Sigma\left(a_{1}^{(\ell)}, a_{2}^{(\ell)}, a_{3}^{(\ell)}\right)$, of $\Sigma(\underline{a})$ for any order $\ell$ with a recurrence relation,

$$
a^{(\ell)}=a^{(\ell-1)}\left(a^{(\ell-1)}+1\right) .
$$

In particular (7) holds for the product of three Seifert invariants $a^{(\ell)}=a_{1}^{(\ell)} a_{2}^{(\ell)} a_{3}^{(\ell)}$.

Definition 5. We define here a sequence of SFH-spheres that we call a primary sequence. Let $p_{i}$ be a prime number which is the $i$-th in the set of positive integers $\mathbb{N}$, e.g. $p_{1}=2, p_{2}=3, \cdots, p_{9}=23, p_{10}=29, \cdots$.

The primary sequence of the SFH-sphere is defined as:

$$
\left\{\Sigma\left(q_{i}, p_{i+1}, p_{i+2}\right) \mid i \in \mathbb{N}\right\}
$$

where $q_{i}=p_{1} \cdots p_{i}$.

Included (for our model) in this sequence, as its first two terms, ordinary three-dimensional spheres $S^{3}$ with Seifert fibration determined by mappings:

$$
h_{p q}: S^{3} \rightarrow S^{2}
$$

which are defined as

$$
h_{p q}\left(z_{1}, z_{2}\right)=z_{1}^{p} / z_{2}^{q}, \quad p, q \in \mathbb{N}
$$

Recall

$$
S^{3}=\left\{\left.\left(z_{1}, z_{2}\right) \in \mathbb{C}^{2}|| z_{1}\right|^{2}+\left|z_{2}\right|^{2}=1\right\},
$$

and

$$
z_{1}^{p} / z_{2}^{q} \in \mathbb{C} \bigcup_{\infty} \cong S^{2} .
$$

The mapping $h_{p q}$, describes the sphere $S^{3}$ with the structure of the Seifert fibration onto an orbifold $S^{2}(p, q)$, isomorphic to the sphere $S^{2}$ with two cone points characterized by the angles $\frac{2 \pi}{p}$ and $\frac{2 \pi}{q}$.

We denote these 3-dimensional spheres (SF-spheres) as $\Sigma(1,1,2), \quad p=1, q=2$ and $\Sigma(1,2,3), \quad p=2, q=3$. 
The Seifert fibration structure in each of these SH-spheres is determined by the numbers $p$ and $q$ but are not unique. Formally, we say that they are special cases of the SFH-spheres. In the notation we use an additional number in the first place of the notation corresponding to an arbitrary regular orbit. This allows us to find the derivative of the above Seifert fibrations $\Sigma(1,1,2), \Sigma(1,2,3)$ and for all elements of the primary sequence using (5).

Now we will form the family of manifolds which belongs to the first-primary SFH-spheres and its derivatives:

Definition 6. Using (5) we define the derivative

$$
\left\{\Sigma^{(\ell)}\left(q_{i-1}, p_{i}, p_{i+1}\right) \mid i \in \overline{0,8}, l \in \overline{0,4}\right\},
$$

where $\overline{0, n}$ is an integer range from 0 to $n$. The spheres $\Sigma(1,1,2)$ and $\Sigma(1,2,3)$ that are included in this family are $i=0,1$ respectively $\left(q_{-1}=q_{0}=p_{0}=1\right)$.

Example: As an example of such derived sequence we present the sequence of the Poincaré homology sphere $\Sigma\left(p_{1}, p_{2}, p_{3}\right)=\Sigma(2,3,5)$.

$$
\begin{gathered}
\Sigma^{(1)}(2,3,5)=\Sigma(2,15,31) \\
\Sigma^{(2)}(2,3,5)=\Sigma(2,465,931) \\
\Sigma^{(3)}(2,3,5)=\Sigma(2,432915,865831) \\
\Sigma^{(4)}(2,3,5)=\Sigma(2,374831227365,749662454731)
\end{gathered}
$$

\subsection{SFH-Spheres Triangulation}

Definition 7. Any smooth compact 3-dimensional manifold $X$ admits a finite closed partition $\tau=\left\{T_{1}, \cdots, T_{N}\right\}$ which comprises closed 3-dimensional simplices (tetrahedra) $T_{i}$ such that $\operatorname{dim}\left(T_{i} \cap T_{j}\right)<3$,

$\forall i, j=1, \cdots, N, i \neq j$. We will call this partition a $c$-triangulation (or canonical triangulation).

The reader can see [8].

We first take $S^{2}$ and extract 3 discs that are tubular neighbourhoods of singular orbits, dividing $S^{2}\left(a_{1}, a_{2}, a_{3}\right)$ on 6 discs: 3 correspond to singular orbits and 3 for regular orbits. See Figure 3.

In Figure 4 we can see an antecedent of a graph.

We start our construction with a singular orbit $S_{i}$. We will see how to represent a regular orbit $h$ in a boundary neighbourhood of a singular orbit

$$
\partial T S_{i}=T^{2} .
$$

Regular orbits are represented by

$$
h=\sigma_{i} m+a_{i} l
$$

where $\sigma_{i}=\frac{a}{a_{i}}$.

Figure 5 shows a $T^{2}$ triangulation represented as a rectangle with edges identified correspondingly to $\sigma_{i}=3$ and $a_{i}=2$ (as a particular case, but it can be generalized to any $\sigma_{i}$ and $a_{i}$ ).

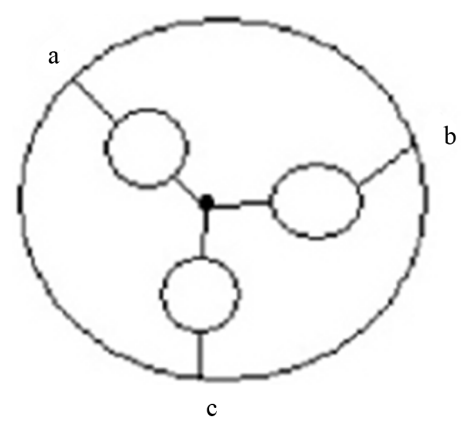

Figure 3. The orbits before removal. 


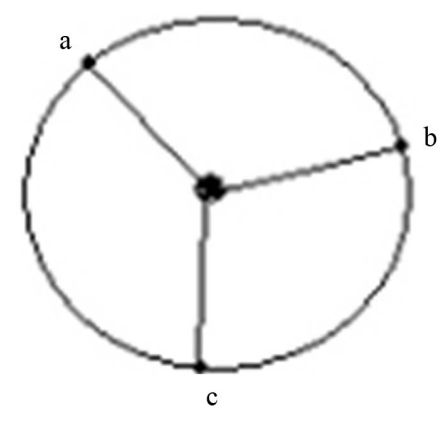

Figure 4. The orbits have been removed.

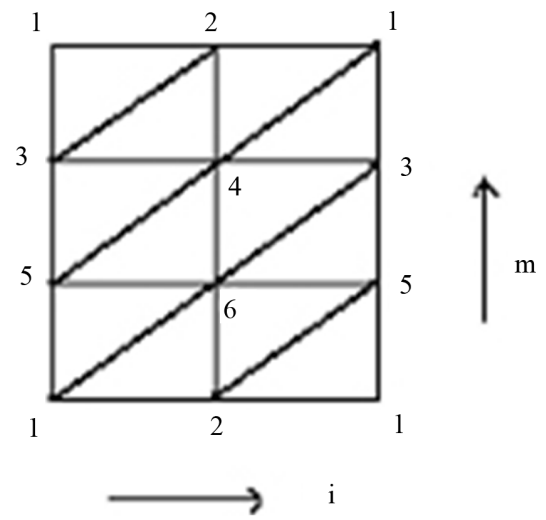

Figure 5. The diagonals marked in bold represent regular fibers.

To obtain tetrahedra from previously made triangulation we build on each one consisting of two adjacent triangles, three tetrahedra. Taking the first two rectangles on the rectangle 1,2,4,3 we do the following tetrahedra with vertices $3,2,4,7,1,2,7,3$ and 1,7,3,8.

The face $(3,4,7,8)$ of the obtained triangular prism $P_{1}$ showed in Figure 6, must be identified (gluing) with the face $(3,4,9,10)$ of a triangular prism $P_{2}$, which rises over 3,4,6,5-rectangle, thereby obtaining a central orbit.

Therefore the number of tetrahedra to triangular a toroidal neighbourhood of a singular orbit is:

$$
N_{(\text {sing) }}=3 a_{1} a_{2} a_{3} .
$$

Now, consider a regular orbit $S$, the representation $h$ of a regular orbit in $\partial T S$ is,

$$
h=l+a m \text {. }
$$

As we can see in Figure 7.

In order to make the complete triangulation including both types of orbits we build on each rectangle consisting of two adjacent triangles, three tetrahedra. See Figure 8.

To have a central orbit we identify the line segment $\overline{b c}$ with $\overline{a d}$. By doing this, the face $(e, c, f)$ of the tetrahedron $d, e, c, f$ is identified with the opposite face. That is why we divide the tetrahedron in two parts. When we identify the faces of these tetrahedra, this induces the subdivision of $a, b, c, e$ and $a, c, d, e$ in each of the two tetrahedra.

\subsection{Cusp Hyperbolic Space Triangulation}

First we need to construct a cusp hyperbolic space with the following process.

Take the 3-dimensional hyperbolic space $\mathbb{H}^{3}$ (each dimension of the 3-dimensional play the role of a spatial section) which is the upper half of $\mathbb{R}^{3}$ with 


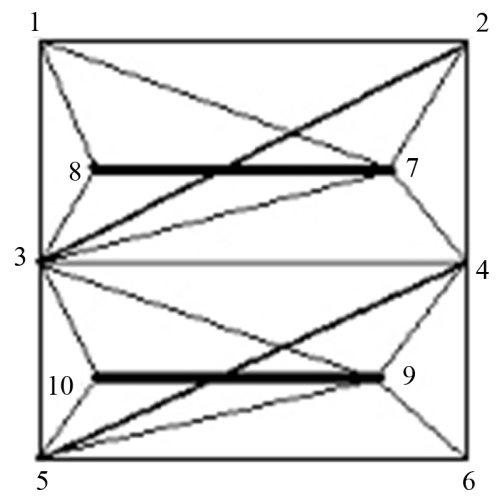

Figure 6. The lines marked in bold represent a central orbit.

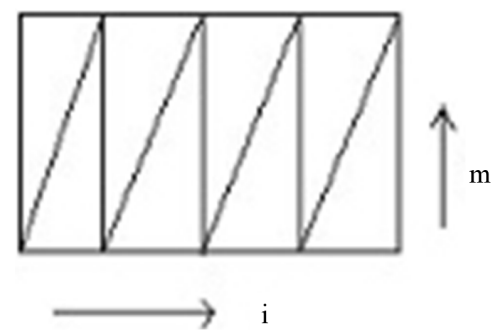

Figure 7. This is a $T^{2}$ triangulation, the diagonals of each rectangle represents a regular fiber $h$.

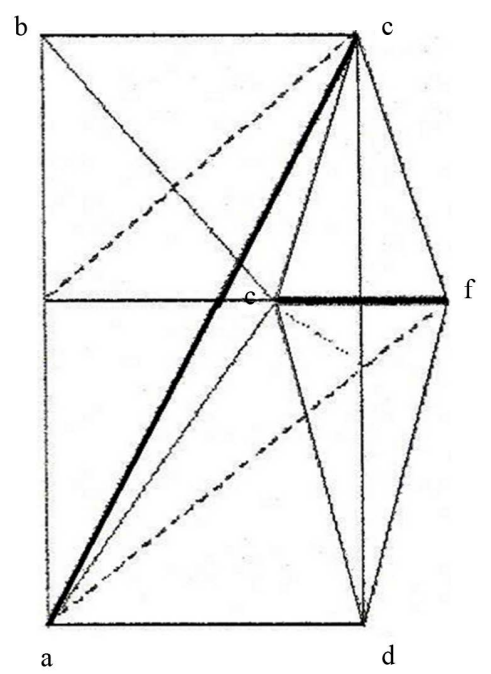

Figure 8. For example, the rectangle $(a, b, c, d)$ to make the tetrahedra, $b, c, e, a, c, d, e$ and $d, e, c, f$.

$$
d s^{2}=\frac{d x^{2}+d y^{2}+d z^{2}}{z^{2}}
$$

and $\partial \mathbb{H}^{3}$ is identified with $\mathbb{C} \cup\{\infty\}$, the isometry group preserving orientation is $\operatorname{PSL}(2, \mathbb{C})$. Hyperbolic 
planes are hemispheres and we have the concept of points at infinity. A fine feature of hyperbolic geometry is the fact that infinite objects can have finite volumes. This is the case of the ideal tetrahedron (one having one or more points at infinity). See [21].

We now follow the Thurston geometrization conjecture [22] to construct our space.

We cut a manifold along essential spheres and tori. We take a connected component of the result of this process, completing spheres with balls and adding the "torus at infinity" in order to obtain the so-called "end" of the manifold. A graphical representation of this is the Figure 9.

Note: If we cut the cusp, we can visualize it as if we cut along a Euclidean plane in the upper half space model and see the previous movements associated to the toroidal boundary. This gives the eigenvalues $\lambda, \mu \in S L(2, \mathbb{C})$. From the last statement we have the abelian group $Z \oplus Z$. This is the acting group in the $\mathbb{H}^{3}$ manifold.

\subsubsection{Cusp Hyperbolic Space}

Here we construct our hyperbolic space with a single cusp and toroidal boundary.

Consider first the cube $M$ which is composed as follows:

Gluing face $A$ with $A^{\prime}$ and $B$ to $B^{\prime}$ (following the direction of the arrows in the cube of the Figure 10), we obtain a cusp hyperbolic space and boundary homeomorphic to a 2-dimensional torus as we can see in Figure 11.

The above construction gives the definition of a cusp.

We need the two-dimensional torus, since in the next section we need to glue the boundary homeomorphically to a torus with either SF-sphere or SFH-sphere.

\subsubsection{Cusp Hyperbolic Space Triangulation}

We describe in this part a triangulation of a cusp hyperbolic space $M$, from a triangulation on the torus.

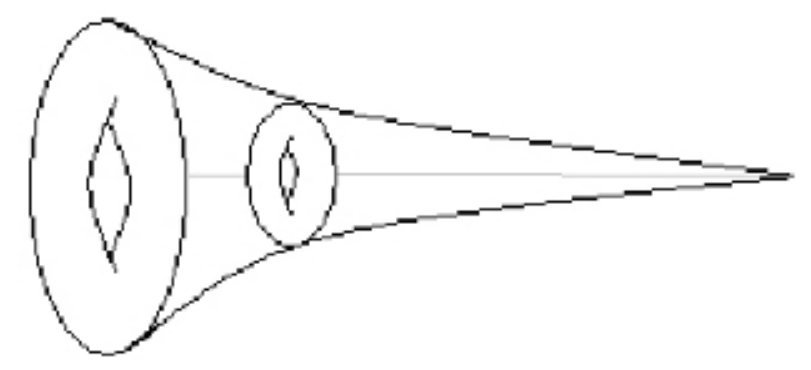

Figure 9. Cusp hyperbolic space.

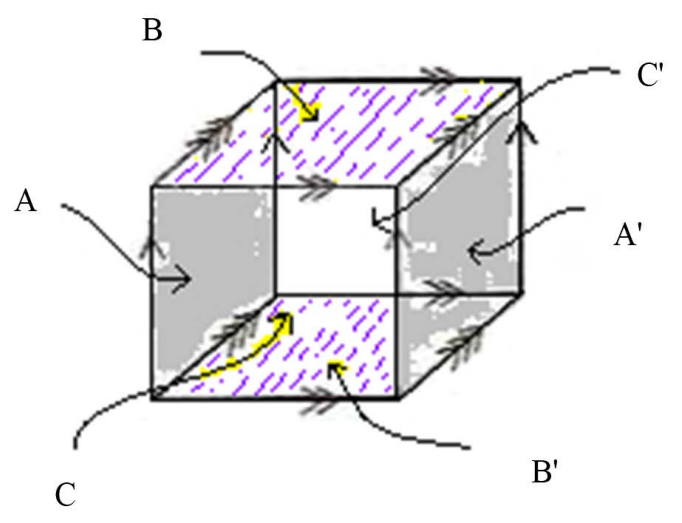

Figure 10. Where the $C$ face has its vertices at infinity for $z \in \mathbb{C}$ and $z=0$; the $C^{\prime}$ face has its vertices in the complex plane $z \in \mathbb{C}$ and $z=r$, for some $r \in \mathbb{R}$ and $r \neq 0$. 


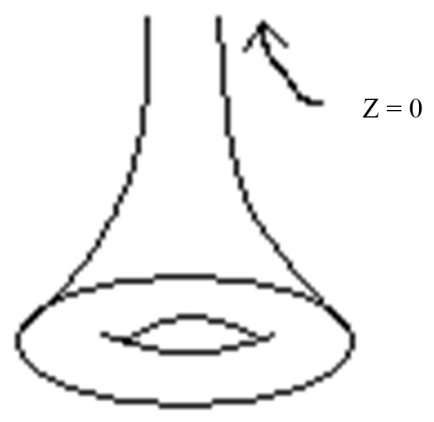

Figure 11. Cusp hyperbolic space with toroidal boundary.

Based on [8] and [9] (with the same ideology) do the following:

Take a partition from a toroidal boundary, with the number of rectangles $a_{1} a_{2} a_{3}$ (where $a_{1} a_{2} a_{3}$ are Seifert hyperbolic invariants).

The details are as follows:

We have first $a_{1} a_{2}=1$ and $a_{3}=1$, i.e. we have two triangles, then we will generalize in order to obtain a formula for the number of generated tetrahedra.

Take the rectangle with vertices $1,2,3,4$, in $C^{\prime}$. We denote by $\eta_{i}$ the points at infinity. Now we take the four points at infinity $\eta_{1}, \eta_{2}, \eta_{3}, \eta_{4} \in C$ (we will call the resulting cube as $Z_{i}$ ) and construct the following tetrahedra $\eta_{1}, \eta_{4}, 1,2 ; \eta_{1}, \eta_{2}, \eta_{3}, 2 ; \eta_{1}, \eta_{3}, \eta_{4}, 2 ; \eta_{4}, \eta_{3}, 2,3 ; 2,3,4, \eta_{4}$ and finally the tetrahedron formed by $1,2,4, \eta_{4}$. Similarly, as in SFH-triangulation we have the number of generated tetrahedra in this case 6 (see Figure 12).

As we need to generalize this process for $a_{1} a_{2}=2$ and $a_{3}=1$, we have, in this case, two glued cubes.

For the cube $Z_{i}$ (showed in Figure 13) we have then 12 tetrahedra, namely $6 a_{1} a_{2} a_{3}$.

Note that by gluing $A$ with $A^{\prime}$ and $B$ with $B^{\prime}$ the tetrahedra of the partition does not generate new vertices, since the figures have symmetrical form. Not having new vertices the gluing will not generate any more tetrahedra.

Generalizing this process for any $a_{1}, a_{2}$ and $a_{3}$, we have:

That is, in Figure 14 we see that for each cube $Z_{i}$ we have 6 tetrahedra. If for example $a_{1} a_{2}=4$ and $a_{3}=3$ then we have 12 cubes, i.e. $12 Z_{i}$ then we will have 72 tetrahedra. The following result is obtained by the above.

$$
H=6 a_{1} a_{2} a_{3} \quad \text { tetrahedra. }
$$

With this we triangulated our cusp hyperbolic space obtaining also the number of generated tetrahedra, given by $H$.

In Section 5 we will construct our model and we will use splice diagrams which are similar to graphs.

\section{Model of a Universe with a Fundamental Interaction}

In our topological model, each fundamental interaction is characterized by the following pair of parameters $(n, \ell), \quad(n, \ell \in \overline{0,4})$. These will be related to an assembly $E_{n h}^{(\ell)}$ of topological spaces composed of Seifert fibered spheres and a cusp hyperbolic space for each singular orbit. With the triangulations obtained in the preceding sections.

Each $(n, \ell)$ interaction is related to an assembly $E_{n h}^{(\ell)}$ of topological spaces $E_{n h}^{(\ell)}(\mathbb{R})$, which are interpreted as a set of allowable 3-spatial sections of the "universe" with one fundamental interaction. See [6] and [27].

We begin by describing the assemblies $E_{n h}^{(\ell)}$. See [10] for the discrete volume.

We take a Seifert fibered sphere (SF-sphere), $\Sigma_{0}^{(\ell)}\left(1, a_{20}^{(\ell)}, a_{30}^{(\ell)}\right)$ of the family

$$
\left\{\Sigma_{n}^{(\ell)}:=\Sigma^{(n-t)}\left(q_{2 n-1}, p_{2 n}, p_{2 n+1}\right) \mid n \in \overline{0,4}, t \in \overline{-4,4}\right\}
$$

which contains two regular and two singular orbits as described in the Subsection 4.2 and we associate the fol- 


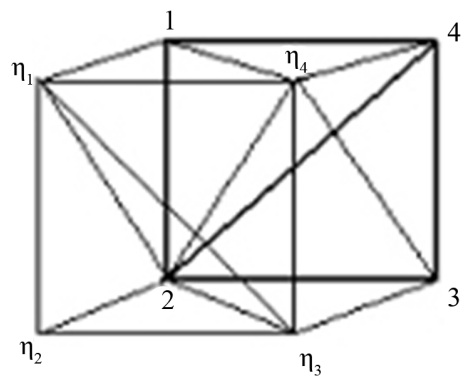

Figure 12. Note that each cube $Z_{i}$ has inside five mixed tetrahedra, i.e. each tetrahedron may have only 2 or 3 or even 1 point at infinity.

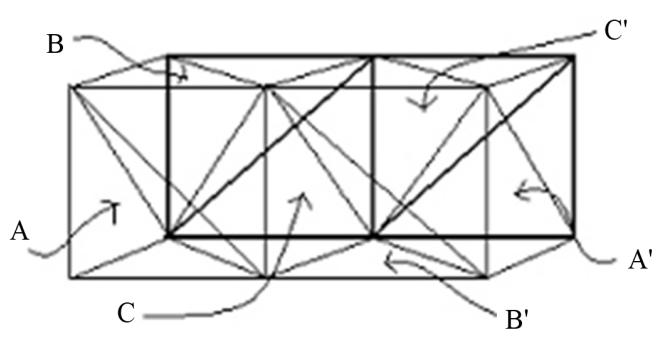

Figure 13. Where $C$ has its vertices at infinity.

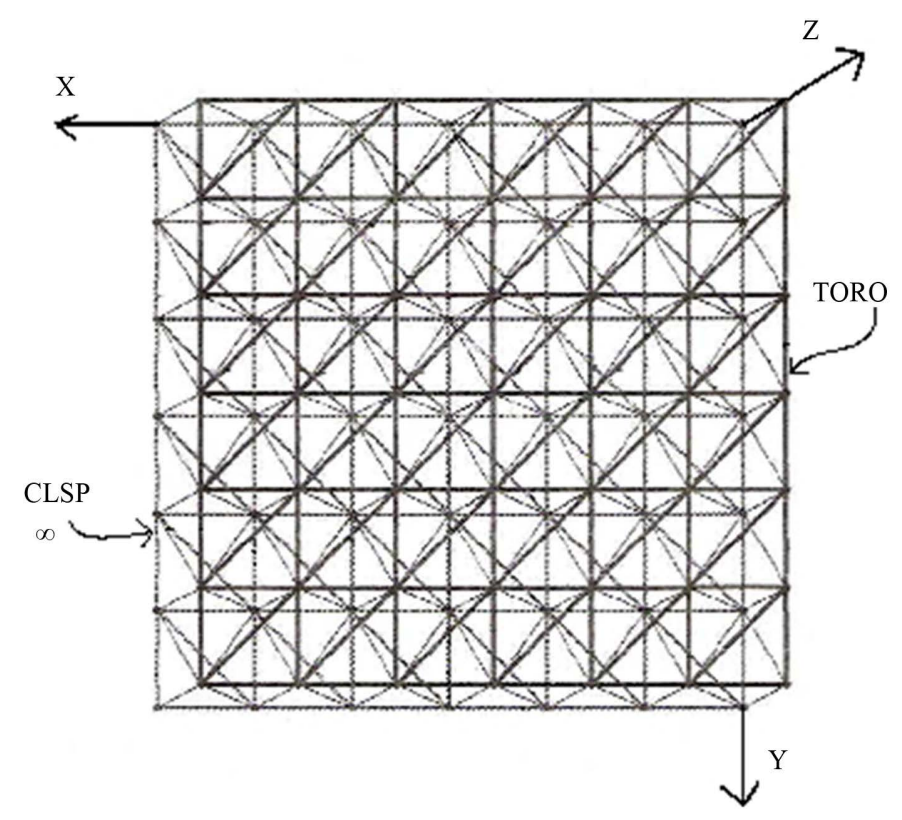

Figure 14. The cusp corresponds to $z=0$ and the back corresponds to $\mathrm{z}=r$ for $\mathrm{z} \in C$ and $r \in R, r \neq 0$.

lowing splice diagram.

$$
\underbrace{a_{20}^{(l)}} \overbrace{}^{a_{30}^{(l)}}
$$

Notation: the second subscript denotes the level $h$, for $n=0$, we have $a_{20}^{(\ell)}$ and $a_{30}^{(\ell)}$. 
Removing the torus neighbourhoods of singular orbits and introducing the torus part of a cusp hyperbolic space by a splice operation (see [17]). Then we have constructed a new manifold denoted by $M_{0 h}^{(\ell)}$

$$
M_{0 h}^{(\ell)}:=h_{0}^{(\ell)} \sum_{a_{1}^{(\ell)} a_{20}^{(\ell)}}^{(\ell)} \Sigma_{a_{30}^{(\ell)} \alpha_{2}^{(\ell)}}^{(\ell)} h_{0}^{(\ell)}
$$

and the corresponding splice diagram.

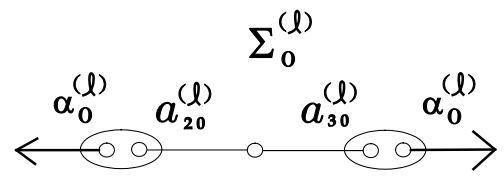

With this process we removed singular orbits and exchanged them by cusp hyperbolic spaces.

Now, consider a SF-sphere $\Sigma_{0}^{(\ell)}\left(1, a_{20}^{(\ell)}, a_{30}^{(\ell)}\right)$ containing two singular and two regular orbits (this is the zero level) and another SF-sphere $\Sigma_{1}^{(\ell)}\left(a_{10}^{(\ell)}, a_{20}^{(\ell)}, a_{30}^{(\ell)}\right)$, containing three singular and three regular orbits (this is level one).

In (18) we have glued two hyperbolic spaces, in order to glue in $\Sigma_{0}^{(\ell)}$. The end of $a_{20}^{(\ell)}$ with $\Sigma_{1}^{(\ell)}$; but in $a_{30}^{(\ell)}$ we must first remove the hyperbolic space $h_{0}^{(\ell)}$ and then we will glue $a_{30}^{(\ell)}$ - with $a_{21}^{(\ell)}$ applying a splice operation. After that, we add two hyperbolic spaces (also level one) in the singular orbits $a_{31}^{(\ell)}$ and $a_{11}^{(\ell)}$ of level one SF-sphere. Here $\alpha_{n}:=a_{1 n}^{(\ell)}, a_{2 n}^{(\ell)}, a_{3 n}^{(\ell)}$.

For the construction of the assembly $\Sigma_{1 h}^{(\ell)}$ we need to define the following manifold:

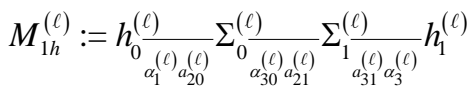

$$
\begin{aligned}
& \mid \begin{array}{l}
a_{11}^{(\ell)} \\
a_{2}^{(\ell)}
\end{array} \\
& h_{1}^{(\ell)}
\end{aligned}
$$

In other words, we are indicating that the splice operation was made in singular orbit $S_{3}$ of $\Sigma_{0}^{(\ell)}$ (which corresponds to $a_{30}^{(\ell)}$ invariant), and $\Sigma_{1}^{(\ell)}$ (corresponding to $a_{21}^{(\ell)}$ invariant) as well as $a_{31}^{(\ell)}$ and $a_{11}^{(\ell)}$ invariants. We remove one toroidal neighbourhood at each singular orbit and it is replaced by the corresponding $h_{1}^{(\ell)}$ space. For this we associate the splice diagram below.

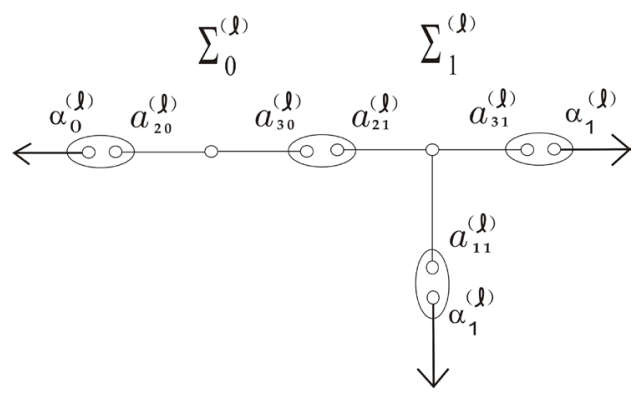

Then the assembly will be defined by:

$$
E_{1 h}^{(\ell)}:=\left\{M_{1 h}^{(\ell)}(R) \mid R \in \overline{0, V_{0 h}^{(\ell)}-2 H_{0}^{(\ell)}}\right\} .
$$

The $M_{1 h}^{(\ell)}(R)$ components are obtained from $M_{0 h}^{(\ell)}$ manifold using the connected sum $R$ times between $M_{0 h}^{(\ell)} \backslash T S_{3}$ sub manifold. Where $T S_{3}$ is the tubular neighbourhood of a singular orbit $S_{3}$ extracted from SFsphere $\Sigma_{0}^{(\ell)}$ using a splice operation. We remove singular orbits of $\Sigma_{1}^{(\ell)}$ and replaced them by a cusp hyperbolic spaces.

Generalizing this idea we get. 


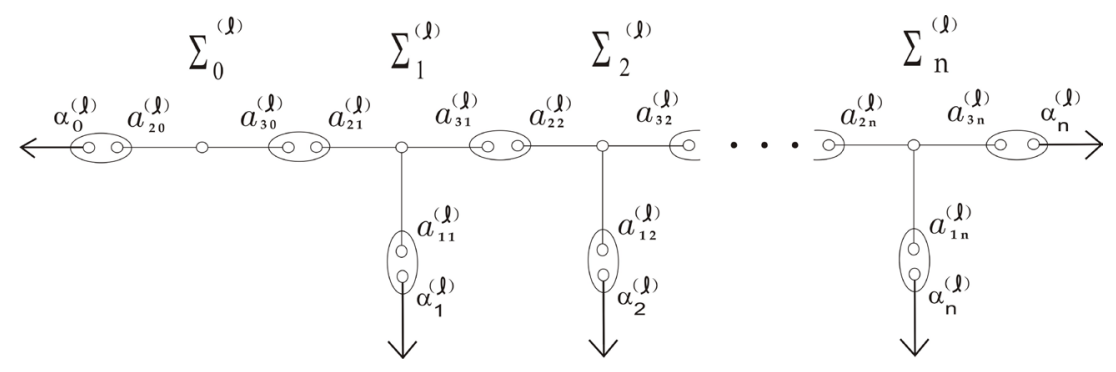

Finally, all singular orbits are removed and replaced by either another SFH-sphere or by a cusp hyperbolic space, fulfilling our goal of avoiding singular orbits (no singular points). See [10] for the discrete volume of this new manifold and a comparison with coupled constants.

\section{Conclusions}

In our construction we remove the tubular neighbourhoods of singular orbits and replace them by a hyperbolic space thus achieving two things. First, avoiding singularities from any space is closer to a physical representation. Usually it is hard to have a physical representation when in theory we have a singularity. Instead, we can imagine singular points as points at infinity. Second, we construct a different space than Efremov and Mitskievich did, introducing Thurston's theory and using the work done by Knesser, Milnor, Jaco, Shalen, Johanson. This space shows an improvement for the calculation of the hierarchy of the coupling constants, i.e. reproducing more closely the exponents of the coupling constants. This is important because the use of hyperbolic spaces instead the local use of hyperbolic geometry make a better approach.

Building the model of the universe with 3-dimensional spatial sections from Seifert fibered homology spheres and cusp hyperbolic spaces provides a description of the evolution at least for the hierarchical approach of the coupling constants by means of topological changes. We also use manifolds which contain a finite volume. Therefore, we can calculate volumes in these spaces. In other words, we have an infinite space with finite volume.

We have created here a new cusp hyperbolic space with fibers. This allows calculating the volume of this particular manifold. Once we obtain how to calculate the volume of each tetrahedra in the space, only what we need is to multiply for the number of tetrahedra contained in the cusp hyperbolic space. All of this is due to the space which was discretized. And this could be used in cosmology theories for calculating the volume density of the space.

As a final comment, we emphasize the importance of the approach presented here for the study of alternative cosmological models. Highlighting an important example, the standard Friedmann Singularity-Free (FSF) theories usually consider more complex structures than the simple spherically symmetric point like a Friedmann singularity (the big bang seed with null dimension and infinite density). Following this path, we point out here a possible application of the topological construction introduced in this paper to cosmological scenarios known as "Cusp Cosmology" which are based on the cusp-like geometries resulting from non-linear wave theory [29].

\section{Acknowledgements}

Thanks to: Ignacio Barradas, Klaus-Peter Schröder, César Caretta, Hugo Garca, Stephanie Dunbar and Lawrence Nash. CONACyT, LAC-INPE, CAPES and PROAP.

\section{References}

[1] Hawking, S. (1988) Wormholes in Spacetime. Physical Review D, 37, 904. http://dx.doi.org/10.1103/PhysRevD.37.904

[2] Weinberg, S. (1989) The Cosmological Constant Problem. Reviews of Modern Physics, 61, 1. http://dx.doi.org/10.1103/RevModPhys.61.1

[3] Horowitz, G.T. (1991) Topology Change in Classical and Quantum Gravity. Classical and Quantum Gravity, 8, 587-601. http://dx.doi.org/10.1088/0264-9381/8/4/007

[4] De Lorenci, V.A., Martin, J., Pinto-Neto, N. and Soares, I.D. (1997) Topology Change in Canonical Quantum Cosmology. 
[5] Kronheimer, P.B. and Mrowka, T.S. (1993) Gauge Theory for Embedded Surfaces. Topology, 32, 773-826. http://dx.doi.org/10.1016/0040-9383(93)90051-V

[6] Efremov, V.N. (1996) Flat Connection Contribution to Topology Changing Amplitudes in an Ensemble of Seifert fibered Homology Spheres. International Journal of Theoretical Physics, 35, 63-68. http://dx.doi.org/10.1007/BF02082934

[7] Efremov, V.N. (1997) Siebenman-Type Cobordisms with Borders and Topology Changes by Quantum Tunnelling. International Journal of Theoretical Physics, 36, 1133-1151. http://dx.doi.org/10.1007/BF02435804

[8] Efremov, V.N. and Mitskievitch, N.V. (2003) Discrete Model of Space-Time in Terms of Inverse Spectra of the $T_{0}$ Alexandroff Topological Spaces.

[9] Efremov, V.N. and Mitskievitch, N.V. (2004) T Discrete Model of Space-Time in Terms of Inverse Spectra of the $T_{0}$ Alexandroff Topological Spaces Opology Changes in Terms of Proper Inverse Spectra of $T_{0}$-Discrete Spaces and Hierarchy of Fundamental Interactions in a Universe Glued Together of Seifert Homologic Spheres. In: Progress in General Relativity and Quantum Cosmology Research, Nova Sci. Publishers.

[10] Mejia, M.E. (2009) Un Modelo del Universo y la Comparación de la Jerarquia de las Constantes de Acoplamiento Experimentales con las Constantes de Acoplamiento del Modelo. Memoria de los extensos del VI Encuentro Participación de la Mujer en la Ciencia, 1, 46-47.

[11] Barrett, J.W. (1995) Quantum Gravity as Topological Quantum Field Theory. Journal of Mathematical Physics, 36, 6161. http://dx.doi.org/10.1063/1.531239

[12] Carlip, S. (2005) Quantum Gravity in 2+1 Dimensions: The Case of a Closed Universe. Living Reviews in Relativity, 8, 1.

[13] Anderson, M., Carlip, S., Ratcliffe, J.G., Surya, S. and Tschantz, S.T. (2004) Peaks in the Hartle-Hawking Wave Function from Sums over Topologies Class. Quantum Gravity, 21, 729. http://dx.doi.org/10.1088/0264-9381/21/2/025

[14] Bytsenko, A.A. and Guimaraes, M.E.X. (2008) Expository Remarks on Three-Dimensional Gravity and Hyperbolic Invariants.

[15] Kneser, H. (1929) Jahresbericht der Deutshen. Mathematiker-Vereinigung, 38, 248-260.

[16] Milnor, J. (1962) A Unique Decomposition Theorem for 3-Manifolds. American Journal of Mathematics, 84, 1-7.

[17] Eisenbud, D. and Newmann, W.D. (1985) Three-Dimensional Link Theory and Invariants of Plane Curve Singularities. Ann. Math. Stud. Princeton University Press.

[18] Johanson, K. (1979) Homotopy Equivalences of 3-Manifolds with Boundaries. Lecture Notes in Math. N 1-761. Springer-Verlag, Berlin.

[19] Jaco, W. and Shalen, P.B. (1979) Seifert Fibered Spaces in 3-Manifolds. American Mathematical Society, 220.

[20] Thurston W. P. (1982) Three-dimensional manifolds, Kleinian groups and hyperbolic geometry. American Mathematical Society. Bulletin. New Series, 6, 357-381.

[21] Thurston, W.P. (1997) The Geometry and Topology of 3-Manifolds. Electronic Version 1.0. http://library.msri.org/books/gt3m/

[22] Thurston, W. P. (1997) Three-Dimensional Geometry and Topology. Vol. 1, Princeton University Press, Princeton.

[23] Jaco, W. (1980) Lectures on 3-Manifold Topology. Regional Conference Series in Mathematics. American Mathematical Soc.

[24] Newmann, W. D. and Zagier, D. (1985) Volumes of Hyperbolic Three-Manifolds. Topology, 24, $307-332$. http://dx.doi.org/10.1016/0040-9383(85)90004-7

[25] Aschenbrenner, M. Friedl, S. and Wilton, H. (2013) 3-Manifold Groups. e-print arXiv:1205.0202 [math.GT].

[26] Rong,Y. (1992) Degree One Maps between Geometric 3-Manifolds. American Mathematical Society, 332, 411-436.

[27] Hernándes, A.M. (2003) Cambios Topológicos y Jerarqua de las Interacciones Fundamentales en un Modelo Cosmológico $T_{0}$-Discreto del Universo Construido de Esferas Homológicas Fibradas de Seifert. Tesis para obtener el grado de maestro en ciencias en la Universidad de Guadalajara.

[28] Milnor, J. (1975) On the 3-Dimensional Brieskorn Manifolds M (p,q,r). http://www.maths.ed.ac.uk/126aar/papersmilnbries.pdf

[29] Rosa, R.R., Strieder, C. and Stalder. D.H. (2012) An Alternative Singularity-Free Cosmological Scenario from Cusp Geometries. AIP Conference Proceedings, 1483, 441-446. http://dx.doi.org/10.1063/1.4756991

[30] Milnor, J. (1958) Review: Norman Steenrod, The Topology of Fibre Bundles. Bulletin of the American Mathematical Society, 64, 202-203. http://dx.doi.org/10.1090/S0002-9904-1958-10211-6 


\section{Appendix}

\section{Orbifold}

Here we take the definition given by [21].

Definition 8. An orbifold $O$ is a space locally modelled on $\mathbb{R}^{n}$ modulus finite group actions. $O$ consists of a Hausdorff space $X_{O}$, with some additional structure. $X_{O}$ has a covering that consists of a collection of open sets $\left\{U_{i}\right\}$ closed under finite intersections. To each $U_{i}$ is associated a finite group $\Gamma_{i}$, an action of $\Gamma_{i}$ on an open subset $\tilde{U}_{i}$ of $R^{n}$ and a homeomorphism $\varphi_{i}: U_{i} \approx \tilde{U}_{i} / \Gamma_{i}$. Whenever $U_{i} \subset U_{j}$, there is an injective homomorphism

$$
f_{i j}: \Gamma_{i} \longmapsto \Gamma_{j}
$$

and an embedding

$$
\varphi_{i j}: \tilde{U}_{i} \longmapsto \tilde{U}_{j}
$$

equivariant with respect to $f_{i j}$ (i.e. for $\left.\gamma \in \Gamma_{i}, \quad \tilde{\varphi}_{i j}(\gamma x)=f_{i j}(\gamma) \tilde{\varphi}_{i j}(x)\right)$ such that the next diagram commutes.

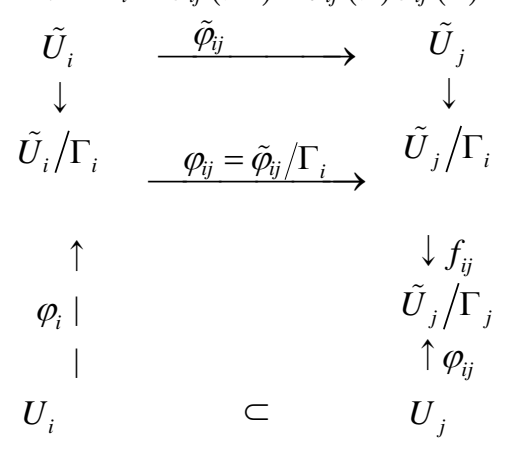

Next we describe some helpful orbifold characteristics.

Definition 9. A point $p$ in a 2-dimensional orbifold $S$ is a cone point with a cone angle $\frac{2 \pi}{p}$, if there exists a neighbourhood around $p$, which is an orbifold diffeomorphic to the quotient orbifold $\mathbb{C} / \mu_{p}$, where $\mu_{p}$ is the group of the p-roots of the unit.

Notation: We will denoted by $M\left(g ; b ; a_{1}, b_{1} ; \cdots ; a_{r}, b_{r}\right)$ a closed orientable Seifert manifold which admits a fibration with basis, where $M^{3} \rightarrow M^{3} / S^{1}=F$ is the Seifert fibration.

Note that the pair $a_{i}, b_{i}$, (where $\operatorname{gcd}\left(a_{i}, b_{i}\right)=1$ for $1 \leq i \leq r$ ) are given by the exceptional fiber. We consider $a_{i}, a_{j}, b_{i}, b_{j}$ are not necessarily relative primes. The last two are given by the exceptional fibers (singular). The genus $g$ characterizes the base space, which is the connected sum of $g$ torus if $g \geq 0$. We have the 2-sphere when $g=0$ and the connected sum of $|g|$ projective planes when $g<0$. The rational

Euler class $e(M)=b+\sum_{i=1}^{r} \frac{b_{i}}{a_{i}} \in \mathbb{Q}$ represents an obstruction to the existence of a section of the fibration, since by reversing the orientation of $M$, reverses also the orientation of the fibers or the base (it does not matter which because $M$ admits an orientation preserving the automorphic mapping of fibers in fibers). We replace the Seifert invariant $\left(g,\left(a_{i}, b_{i}\right)\right)$ by $\left(g,\left(a_{i},-b_{i}\right)\right)$ then $e(-M)=-e(M)$. If $r>0$, changing some $b_{i}$ to $b_{i}+a_{i} \beta$, then we have $b=0$. Henceforth, we will assume that $b=0$ when $g=0$, denoting such manifold by $M\left(a_{1}, b_{1} ; \cdots ; a_{r}, b_{r}\right)$.

Like homology spheres are a particular case of SFM, here is the follow definition.

Definition 10. Homology spheres. Let $a_{1}, \cdots, a_{n}$ be co prime integers with $n \geq 3$ and each $a_{i}>2$. Then there is a 3-SFM, with a Seifert invariant that has the form

with

$$
\left(0:\left(a_{1}, b_{1}\right), \cdots,\left(a_{n}, b_{n}\right)\right)
$$

$$
\sum_{i=1}^{n} b_{i} a_{1} \cdots a_{i} \cdots a_{n}=1 .
$$


This manifold is denoted by $\Sigma\left(a_{1}, \cdots, a_{n}\right)$ and it is a homology sphere.

By $\hat{a}_{i}$ we denote that $a_{i}$ is not there.

For any oriented Seifert fibered homology sphere $\Sigma \nsubseteq S^{3}$. There is a unique n-tuple $\left(a_{1}, \cdots, a_{n}\right)$ with single orientation sign \pm , such that $\Sigma \cong \pm \Sigma\left(a_{1}, \cdots, a_{n}\right)$. This orientation preserves homeomorphisms. This homeomorphism can be chosen to preserve the Seifert fiber structure. As an additional reference on fiber bundles we refer reader to might look [30].

\section{Topological Description of Homology Spheres}

Let $F_{0}=S^{2}-\operatorname{int}\left(D_{1}^{2} \cup \cdots \cup D_{n}^{2}\right)$ be a $n$-fold of the 2-punctured sphere, then $\Sigma=\Sigma\left(a_{1}, \cdots, a_{n}\right)$ can be characterized by an oriented closed 3-manifold in which we can immerse pairwise disjoint solid torus $\left(D^{2} \times S^{1}\right), \cdots,\left(D^{2} \times S^{1}\right)$ such that:

1) If $\Sigma_{0}=\Sigma-i n t\left(\left(D^{\underline{2}} \times S^{1}\right)_{1} \cup \cdots \cup\left(D^{2} \times S^{1}\right)_{n}\right)$

then, exists a fibration $\pi: \Sigma_{0} \rightarrow F_{0}$ with fiber $S^{1}$, so $\Sigma_{0} \cong F_{0} \times S^{1}$.

2) If $R \subset \Sigma_{0}$ is a section of $\pi$ and for $i=1,2, \cdots, n$.

$$
\begin{aligned}
& Q_{i}=-\partial R \cap\left(D^{2} \times S^{1}\right)_{i} \\
& H_{i}=\text { typical fiber of } \pi \text { in } \partial\left(D^{2} \times S^{1}\right)_{i}
\end{aligned}
$$

then $a_{i} Q_{i}+b_{i} H_{i}$ is null homologous to $\left(D^{2} \times S^{1}\right)$, where each $b_{i}$ satisfies (20).

We remark that $(0.20)$ determines $\beta_{i}\left(\operatorname{moda}_{i}\right)$ and $\operatorname{gcd}\left(a_{i}, \beta_{i}\right)=1$. In a geometric description we have that

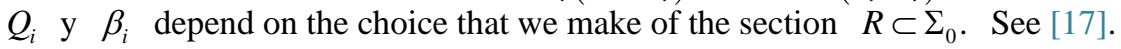

